\title{
ON THE FUNDAMENTAL GROUP OF A VARIETY WITH QUOTIENT SINGULARITIES
}

\author{
INDRANIL BISWAS AND AMIT HOGADI
}

\begin{abstract}
Let $k$ be a field, and let $\pi: \widetilde{X} \longrightarrow X$ be a proper birational morphism of irreducible $k$-varieties, where $\widetilde{X}$ is smooth and $X$ has at worst quotient singularities. When the characteristic of $k$ is zero, a theorem of Kollár in Ko1 says that $\pi$ induces an isomorphism of étale fundamental groups. We give a proof of this result which works for all characteristics. As an application, we prove that for a smooth projective irreducible surface $X$ over an algebraically closed field $k$, the étale fundamental group of the Hilbert scheme of $n$ points of $X$, where $n>1$, is canonically isomorphic to the abelianization of the étale fundamental group of $X$. Kollár has pointed out how the proof of the first result can be extended to cover the case of quotients by finite group schemes.
\end{abstract}

\section{INTRODUCTION}

Following is the main result proved here:

Theorem 1.1. Let $k$ be any field and $X / k$ a connected variety which étale locally can be expressed as a quotient of a smooth variety by the action of a finite group of automorphisms of it. Let

$$
\pi: \widetilde{X} \longrightarrow X
$$

be a proper birational morphism with $\widetilde{X}$ smooth and connected. Then for any geometric point $\widetilde{x}_{0}$ of $\widetilde{X}$, the induced homomorphism

$$
\pi_{*}: \pi_{1}^{e t}\left(\widetilde{X}, \widetilde{x}_{0}\right) \longrightarrow \pi_{1}^{e t}\left(X, \pi\left(\widetilde{x}_{0}\right)\right)
$$

is an isomorphism.

When the characteristic of $k$ is zero, this is a theorem of Kollár [Ko1, p. 203, Theorem (7.5.2)]. Theorem 1.1 is proved in Section 3, János Kollár has pointed out, [Ko2, that the proof of Theorem 1.1] can be extended to establish a stronger result that addresses the more general situation of quotient of smooth varieties by finite group schemes (see Theorem 3.5).

Let $X$ be a connected smooth projective surface over an algebraically closed field $k$. For any positive integer $n$, let $\operatorname{Hilb}^{n}(X)$ denote the Hilbert scheme of

2000 Mathematics Subject Classification. 14C05, 14F35, 4L30.

Key words and phrases. Étale fundamental group, quotient singularity, symmetric product, abelianization, Dold-Thom theorem, Hilbert scheme. 
$n$ points on $X$. This $\operatorname{Hilb}^{n}(X)$ is an irreducible smooth projective variety of dimension $2 n$ ([Fo, p. 517, Proposition 2.3] and [Fo, p. 517, Theorem 2.4]). The motivation of this paper was to calculate the fundamental group of $\operatorname{Hilb}^{n}(X)$. When $k$ is of characteristic zero, the fundamental group of $\operatorname{Hilb}^{n}(X)$, where $n$ is at least two, is already known to be isomorphic to the abelianization of the fundamental group of $X$. The proof of this (for characteristic zero) consists of two steps. First, one shows that the fundamental group of $\operatorname{Sym}^{n}(X)$, the $n$-th symmetric product of $X$, is the abelianization of that of $X$ provided $n>1$. This is in fact a purely topological result (cf. Theorem 5.1 and Example 5.2 of [KT]; see also [DT]). Now $\operatorname{Hilb}^{n}(X)$ is a desingularization of $\operatorname{Sym}^{n}(X)$ [Fo, p. 518, Corollary 2.6]. The second step of the proof is provided by the above mentioned result of Kollár in [Ko1].

The following is an analogue of the topological result mentioned earlier.

Theorem 1.2. Let $k$ be an algebraically closed field, and let $X / k$ be a variety which is integral and proper. For fixed $n>1$, let $x_{0}$ and $\underline{x}$ denote geometric points of $X$ and $\operatorname{Sym}^{n}(X)$ respectively. Then $\pi_{1}^{\text {et }}\left(\operatorname{Sym}^{n}(X), \underline{x}\right)$ is canonically isomorphic to the abelianization of $\pi_{1}^{e t}\left(X, x_{0}\right)$.

Theorem 1.2 is proved at the end of Section 4 .

Putting Theorem 1.1 and Theorem 1.2 together, one gets the following.

Corollary 1.3. Let $X$ be a connected smooth projective surface over an algebraically closed field $k$. For any $n>1$, the fundamental group of $\operatorname{Hilb}^{n}(X)$ is canonically isomorphic to the abelianization of the fundamental group of $X$.

Acknowledgements. We thank János Kollár for his comments on an initial version. Subsequently, he pointed out how Theorem 1.1 could be generalized Ko2]. We are very grateful to him for this. We are very grateful to the referee for helpful comments. The proof of Theorem 3.5 was substantially simplified by the referee. The first-named author is supported by the J. C. Bose Fellowship.

\section{Fundamental group of a Desingularization of the COARSE MODULI SPACE}

The goal of this section is to give a description of the fundamental group of a desingularization of the coarse moduli space of a smooth Deligne-Mumford stack (see Lemma 2.6).

We start by recalling some notation and state the main result, [No, Section 7], of Noohi in [No]. Let $\mathcal{X} / k$ be a connected separated Deligne-Mumford stack of finite type over a field $k$. Let

$$
\pi: \mathcal{X} \longrightarrow X
$$

be its coarse moduli space. For every geometric point $q: \operatorname{Spec}(L) \longrightarrow \mathcal{X}$, one has an induced map

$$
q:\left[\operatorname{Spec}(L) / G_{q}\right] \longrightarrow \mathcal{X}
$$


where $G_{q}$ is the isotropy group at $q$. When it is necessary to make an explicit reference to $\mathcal{X}$, we will write $G_{q, \mathcal{X}}$ instead of $G_{q}$. Since the étale fundamental group of $\left[\operatorname{Spec}(L) / G_{q}\right]$ at the canonical point $\operatorname{Spec}(L) \longrightarrow\left[\operatorname{Spec}(L) / G_{q}\right]$ is $G_{q}$, we have an induced homomorphism

$$
\phi_{q}: G_{q} \longrightarrow \pi_{1}^{e t}(\mathcal{X}, q) .
$$

The stack $\mathcal{X}$ being connected, for any other geometric point $p$, one has an isomorphism

$$
\pi_{1}^{e t}(\mathcal{X}, p) \stackrel{\sim}{\longrightarrow} \pi_{1}^{e t}(\mathcal{X}, q)
$$

resulting in an equivalence class homomorphisms

$$
\phi_{q}^{p}: G_{q} \longrightarrow \pi_{1}^{e t}(\mathcal{X}, p),
$$

where two homomorphisms are equivalent if they differ by an inner automorphism of $\pi_{1}^{e t}(\mathcal{X}, p)$. In other words, we get a homomorphism $\phi_{q}^{p}$ well defined up to conjugation.

Let $N_{p}$ denote the closed normal subgroup of $\pi_{1}^{e t}(\mathcal{X}, p)$ generated by the images of $\phi_{q}^{p}$ for all geometric points $q$ of $\mathcal{X}$.

Theorem 2.1 ([No]). Let $\mathcal{X}, \pi$ and $X$ be as above. For a geometric point $p$ of $\mathcal{X}$, the corresponding point of $X$ is also denoted by $p$. There is a natural sequence of groups

$$
1 \longrightarrow N_{p} \longrightarrow \pi_{1}^{e t}(\mathcal{X}, p) \longrightarrow \pi_{1}^{e t}(X, p) \longrightarrow 1
$$

which is exact.

Remark 2.2. We continue with the above notation. Let $\mathcal{W} \longrightarrow \mathcal{X}$ be a Galois étale cover such that $N_{p}$ acts trivially on $\mathcal{W}$. Then the above theorem of Noohi says that $\mathcal{W}$ is induced by a Galois étale cover of $X$. It follows from property (M4) in [No, p. 83] of the coarse moduli space functor that this étale cover of $X$ is nothing but the coarse moduli space of $\mathcal{W}$. This coarse moduli space will be denoted by $W$. Thus the following conclusion may be deduced from the above theorem of Noohi: For a Galois étale cover $\mathcal{W} \longrightarrow \mathcal{X}$, the induced map $W \longrightarrow X$ on the coarse moduli spaces is étale if and only if $\mathcal{W}$ is the pullback of $W$ to $\mathcal{X}$.

For our purpose, we would also like to have an explicit relation between the fundamental group of a smooth Deligne-Mumford stack $\mathcal{X}$ and that of a desingularization of its coarse moduli space, whenever such a desingularization exists. For that purpose we set up the following notation.

Notation 2.3. For a smooth Deligne-Mumford stack $\mathcal{X}$, let $E T(\mathcal{X})$ denote the category of finite étale covers of $\mathcal{X}$. Let $\mathbb{B}_{\mathcal{X}}$ be the set of all isomorphism classes of 1-morphisms $f: \mathcal{Y} \longrightarrow \mathcal{X}$, where $\mathcal{Y}$ is a connected normal Deligne-Mumford stack, and $f$ is proper, representable and birational. Now consider the set $\Sigma_{\mathcal{X}}$ of 
all pairs of the form $(\mathcal{Y} \stackrel{f}{\rightarrow} \mathcal{X}, q)$, where $f \in \mathbb{B}_{\mathcal{X}}$, and $q$ is a codimension one geometric point of $\mathcal{Y}$. For any such pair, we have a homomorphism

$$
\phi_{q, \mathcal{X}}: G_{q, \mathcal{Y}} \longrightarrow \pi_{1}^{e t}\left(\mathcal{X}, x_{0}\right)
$$

defined by the composition

$$
G_{q, \mathcal{Y}} \longrightarrow G_{p, \mathcal{X}} \stackrel{\phi_{p}}{\longrightarrow} \pi_{1}^{e t}(\mathcal{X}, p)
$$

where $p=f(q)$ and $\phi_{p}$ is as in (2.1). We note that the homomorphism $\phi_{q, \mathcal{X}}$ in (2.3) is well defined up to an inner automorphism of $\pi_{1}^{e t}\left(\mathcal{X}, x_{0}\right)$ (this was explained earlier in (2.2) $)$. For a geometric point $x_{0}$ of $\mathcal{X}$, let $\widetilde{N}_{x_{0}}$ denote the closed normal subgroup generated by the union of the images of $\phi_{q, \mathcal{X}}$ for all pairs $(\mathcal{Y}, q)$ in $\Sigma_{\mathcal{X}}$.

Lemma 2.4. Let $\mathcal{Z}$ be a regular algebraic stack and $i: U \hookrightarrow \mathcal{Z}$ be a dense open subset. Let

$$
i^{*}: \operatorname{ET}(\mathcal{Z}) \longrightarrow \operatorname{ET}(U)
$$

be the pullback functor. Then the following hold:

(1) $i^{*}$ is fully faithful.

(2) If the complement of $U$ in $\mathcal{Z}$ has codimension at least two, then $i^{*}$ is an equivalence of categories.

Proof. (1) Let $f_{i}: W_{i} \longrightarrow \mathcal{Z}, i=1,2$, be two finite étale covers, and denote by $W_{i U}$ their restrictions to $U$. We need to show that the map

$$
\beta: \operatorname{Hom}_{\mathcal{Z}}\left(W_{1}, W_{2}\right) \longrightarrow \operatorname{Hom}_{U}\left(W_{1 U}, W_{2 U}\right)
$$

is a bijection. Since the maps $f_{i}$ are separated, it is easy to see that the map $\beta$ in (2.4) is injective. Now take any $h \in \operatorname{Hom}_{U}\left(W_{1 U}, W_{2 U}\right)$. Let $\Gamma \subset W_{1} \times_{\mathcal{Z}} W_{2}$ be the closure of the graph of $h$. It is enough to show that $\Gamma$ is a graph of a morphism, i.e., the projection $\varpi: \Gamma \longrightarrow W_{1}$ is an isomorphism. That $\varpi$ is an isomorphism can be shown locally on $\mathcal{Z}$. Thus we may assume $\mathcal{Z}$ and $W_{i}$ 's are schemes. Moreover, by further étale base change, we may assume $W_{i}$ 's are trivial étale covers. In this case, the claim is obvious.

(2) By (1), we only need to show essential surjectivity of $i^{*}$. In other words, we have to show that any finite étale cover $T \longrightarrow U$ extends uniquely to a finite étale cover $T^{\prime} \longrightarrow \mathcal{Z}$. The claimed uniqueness and descent allows us to prove the claim étale (or even smooth) locally on $\mathcal{Z}$. Thus we may base extend to an atlas of $\mathcal{Z}$ and assume that $\mathcal{Z}$ is a scheme. In this case, by [EGA, 8.12.6], we can extend $T \longrightarrow U$ to a finite map $T^{\prime} \longrightarrow \mathcal{Z}$. By normalizing $T^{\prime}$, we may further assume that $T^{\prime}$ is normal. By purity of branched locus (see [SGA, p. 212, X.3.1]) it follows that $T^{\prime} \longrightarrow \mathcal{Z}$ is finite étale.

Lemma 2.5. Let $\{f: \mathcal{Y} \longrightarrow \mathcal{X}\} \in \mathbb{B}_{\mathcal{X}}$. Let $\mathcal{Y}_{\text {sm }}$ denote the smooth locus of $\mathcal{Y}$. Then

$$
f_{E T}^{*}: E T(\mathcal{X}) \longrightarrow E T\left(\mathcal{Y}_{s m}\right)
$$


is an equivalence of categories.

Proof. Since $\mathcal{X}$ is smooth and $f$ is proper and birational, there exists an open substack $U \subset \mathcal{X}$ whose complement has codimension at least two and $f^{-1}(U) \stackrel{f}{\longrightarrow} U$ is an isomorphism. Thus $f^{-1}(U) \cap \mathcal{Y}_{s m}$ is an open subset of $\mathcal{Y}_{s m}$ which maps isomorphically onto an open subset of $\mathcal{X}$. By Lemma 2.4(1),

$$
\operatorname{ET}(\mathcal{X}) \longrightarrow \operatorname{ET}\left(\mathcal{Y}_{s m}\right)
$$

is fully faithful, and we only need to show it is essentially surjective.

Now $E T(U) \longrightarrow E T\left(f^{-1}(U)\right)$ is an equivalence of categories. As $\mathcal{Y}$ is normal, it follows that the complement of $\mathcal{Y}_{s m}$ in $\mathcal{Y}$ has codimension at least two. Therefore, the composition

$$
E T(\mathcal{X}) \stackrel{\text { purity }}{\longrightarrow} \operatorname{ET}(U) \longrightarrow E T\left(f^{-1}(U)\right) \stackrel{\text { purity }}{\longrightarrow} \operatorname{ET}\left(\mathcal{Y}_{s m} \cap f^{-1}(U)\right)
$$

is an equivalence of categories. Here the equivalences labelled by "purity" follow from Lemma 2.4 (2). Thus if $\mathcal{W} \longrightarrow \mathcal{Y}_{s m}$ is a finite étale cover, then its restriction to $\mathcal{Y}_{s m} \cap f^{-1}(U)$ is a pullback of a finite étale cover of $\mathcal{W}^{\prime} \longrightarrow \mathcal{X}$. By Lemma 2.4(1), this pullback and $\mathcal{W}$ must be isomorphic, because they are isomorphic after restricting to a dense open subset. This shows that $\operatorname{ET}(\mathcal{X}) \longrightarrow E T\left(\mathcal{Y}_{s m}\right)$ is essentially surjective, as required.

For $\mathcal{Y}_{1}, \mathcal{Y}_{2} \in \mathbb{B}_{\mathcal{X}}$, we denote the normalization of the unique dominant irreducible component of $\mathcal{Y}_{1} \times \mathcal{X} \mathcal{Y}_{2}$ by $\left(\mathcal{Y}_{1} \times \mathcal{X} \mathcal{Y}_{2}\right)^{0}$.

Lemma 2.6. Let $\mathcal{X} / k$ be a smooth connected Deligne-Mumford stack, and let $\mathcal{X} \stackrel{q}{\longrightarrow} X$ be its coarse moduli space. Let $\pi: \tilde{X} \longrightarrow X$ be a proper birational morphism with $\tilde{X} / k$ smooth. Let $x_{0}$ be a geometric point of $\mathcal{X}$ whose image in $X$ lies in the open subset over which $\pi$ is an isomorphism, thus $x_{0}$ also defines a geometric point of $\widetilde{X}$. Then there is a natural short exact sequence of groups

$$
1 \longrightarrow \tilde{N}_{x_{0}} \longrightarrow \pi_{1}^{e t}\left(\mathcal{X}, x_{0}\right) \longrightarrow \pi_{1}^{e t}\left(\tilde{X}, x_{0}\right) \longrightarrow 1 \text {. }
$$

Proof. We will prove this in three steps.

Step(1): We first observe that there exists a canonical functor

$$
\operatorname{ET}(\tilde{X}) \longrightarrow \operatorname{ET}(\mathcal{X})
$$

defined as follows. Given a finite étale cover of $\widetilde{X}$, restrict it to $\pi^{-1}\left(X_{s m}\right)$, where $X_{s m} \subset X$ is the smooth locus. Since $X_{s m}$ is by definition smooth, this cover descends to define a finite étale cover of $X_{s m}$ and hence of $\alpha^{-1}\left(X_{s m}\right)$, where

$$
\alpha: \mathcal{X} \longrightarrow X
$$

is the coarse moduli map. But $\alpha^{-1}\left(X_{s m}\right)$ is an open sub-stack of $\mathcal{X}$ whose complement has codimension at least two, and hence by purity, this extends uniquely to give a finite étale cover of $\mathcal{X}$. It is also easy to see that the functor 
$E T(\widetilde{X}) \longrightarrow E T(\mathcal{X})$ defined this way sends connected étale covers to connected étale covers. Therefore, we get a surjective group homomorphism

$$
\pi_{1}^{e t}\left(\mathcal{X}, x_{0}\right) \longrightarrow \pi_{1}^{e t}\left(\widetilde{X}, x_{0}\right) .
$$

Step(2): We now claim that $\widetilde{N}_{x_{0}}$ is in the kernel of the homomorphism in (2.5).

To prove this claim, given any pair $\left(\mathcal{Y}, y_{0}\right) \in \Sigma_{\mathcal{X}}$, define

$$
\mathcal{Y}_{1}:=\left(\mathcal{Y} \times_{X} \tilde{X}\right)^{0}
$$

and let $y_{1}$ be the unique lift of $y_{0}$ in $\mathcal{Y}_{1}$. Clearly we have $\mathcal{Y}_{1} \in \mathbb{B}_{\mathcal{X}}$. Since $\mathcal{Y}$ and $\mathcal{Y}_{1}$ are normal, the map $\mathcal{Y}_{1} \longrightarrow \mathcal{Y}$ is an isomorphism over codimension one points of $\mathcal{Y}$. Consequently, the isotropy groups at $y_{0}$ and $y_{1}$ coincide. By replacing $\mathcal{Y}$ by $\mathcal{Y}_{1}$, we may assume, without any loss of any generality, that we have a 1-morphism $g: \mathcal{Y} \longrightarrow \widetilde{X}$ such that the following diagram is commutative

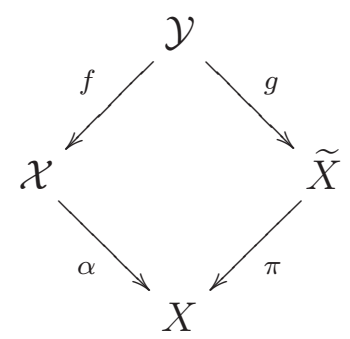

Let $y_{0}^{\prime}$ be a geometric point of $\mathcal{Y}$ such that $g\left(y_{0}^{\prime}\right)=x_{0}$. Since $\tilde{X}$ is a variety, the composition homomorphism $G_{y_{0}} \longrightarrow \pi_{1}^{e t}\left(\mathcal{Y}, y_{0}^{\prime}\right) \longrightarrow \pi_{1}^{e t}\left(\tilde{X}, x_{0}\right)$ is trivial. This proves the claim.

Step(3): To prove the theorem it remains to show that the kernel of the homomorphism in (2.5) is contained in $\widetilde{N}_{x_{0}}$.

Take any $(\mathcal{Y}, q) \in \Sigma_{\mathcal{X}}$. For the geometric point $q$, represented by $\operatorname{Spec}\left(k_{q}\right) \longrightarrow$ $\mathcal{Y}$, with $k_{q}$ separably closed, there is a natural map

$$
B G_{q}=\left[\operatorname{Spec}\left(k_{q}\right) / G_{q}\right] \longrightarrow \mathcal{Y}
$$

and hence we get a map $B G_{q} \longrightarrow \mathcal{X}$. Let $E T(\mathcal{X})^{\prime}$ be the full subcategory of $E T(\mathcal{X})$ consisting of finite étale covers $W \longrightarrow \mathcal{X}$ which have the property that for any $(\mathcal{Y}, q) \in \Sigma_{\mathcal{X}}$, the pullback of $W \times{ }_{\mathcal{X}} B G_{q} \longrightarrow B G_{q}$ is a trivial cover (this means that each connected component of it is isomorphic to $B G_{q}$ ).

If $W \longrightarrow \mathcal{X}$ is a Galois cover, then the above condition is equivalent to the condition that the image of $G_{q}$ in $\pi_{1}^{e t}\left(\mathcal{X}, x_{0}\right)$ acts trivially on $W$. Note however that the kernel of the homomorphism $\pi_{1}^{e t}\left(\mathcal{X}, x_{0}\right) \longrightarrow \operatorname{Gal}(W / \mathcal{X})$ is a normal subgroup. And hence the condition that all $G_{q}$ 's are contained in the normal subgroup is equivalent to the condition that $\widetilde{N}_{x_{0}}$ is contained in the kernel. Thus, 
$E T(\mathcal{X})^{\prime}$, together with the fiber functor defined by the point $x_{0}$, is a Galois category whose associated Galois group is $\pi_{1}^{e t}\left(\mathcal{X}, x_{0}\right) / \widetilde{N}_{x_{0}}$.

Consequently, in order to prove that the kernel is contained in $\widetilde{N}_{x_{0}}$, it suffices to show that the pullback functor defined in Step (1)

$$
\operatorname{ET}(\tilde{X}) \longrightarrow E T(\mathcal{X})
$$

induces an equivalence of $E T(\mathcal{X})$ with $E T(\mathcal{X})^{\prime}$. As, observed before, the pullback functor is fully faithful. Thus it remains to show that its essential image coincides with $E T(\mathcal{X})^{\prime}$, in other words, any étale cover in $E T(\mathcal{X})^{\prime}$ is a pullback of an étale cover of $\widetilde{X}$. Define

$$
\mathcal{Y}:=\left(\mathcal{X} \times_{X} \tilde{X}\right)^{0} \text {. }
$$

Then we have $g: \mathcal{Y} \longrightarrow \widetilde{X}$ and a commutative diagram as in (2.6). Let

$$
h: \mathcal{Y} \longrightarrow Y
$$

be the coarse moduli space. The morphism $g$ as in (2.6) factors through $h$ by the definition of a coarse moduli space.

Now, let $W \longrightarrow \mathcal{X}$ be a Galois étale cover contained in $\operatorname{ET}(\mathcal{X})^{\prime}$. Let

$$
W_{\mathcal{Y}}=W \times \mathcal{X} \mathcal{Y} \longrightarrow \mathcal{Y}
$$

be the Galois étale cover. Consider the induced map

$$
\xi: W^{\prime} \longrightarrow Y
$$

on the coarse moduli spaces, where $W^{\prime}$ is the coarse moduli space of $W$. We note that $\xi$ is a finite map. Let $V \subset Y$ be the maximal open subset of $Y$ over which this map is étale. By Lemma 2.7 below, and definition of $\operatorname{Et}(\mathcal{X})^{\prime}$, this subset $V$ contains all codimension one points of $Y$. By purity, the Galois étale cover $\left.W^{\prime}\right|_{V} \longrightarrow V$ extends to a Galois étale cover of the smooth locus of $Y$ and hence also to a Galois étale cover of $\widetilde{X}$ by Lemma 2.5.

Lemma 2.7. Let $\mathcal{Y} / k$ be an orbifold and $\mathcal{W} \longrightarrow \mathcal{Y}$ a finite étale cover. The coarse moduli spaces for $\mathcal{Y}$ and $\mathcal{W}$ will be denoted by $Y$ and $W$ respectively. Let $W \longrightarrow Y$ be the map induced by the étale cover. Let $q$ be a geometric point of $Y$ represented by $\operatorname{Spec}\left(k_{q}\right) \longrightarrow \mathcal{Y}$, where $k_{q}$ is separably closed. Let

$$
B G_{q}=\left[\operatorname{Spec}\left(k_{q}\right) / G_{q}\right] \longrightarrow \mathcal{Y}
$$

be the induced map. Then the following are equivalent:

(1) $\mathcal{W} \times y B G_{q} \longrightarrow B G_{q}$ is a trivial étale cover (i.e., isomorphic to the projection from a disjoint union of copies of $\left.B G_{q}\right)$.

(2) $W \longrightarrow Y$ is étale over the image of $q$ in $Y$.

Proof. Without loss of generality, we first replace $k$ by a finite étale extension, and assume that the finite group scheme $G_{q}$ is actually a discrete group. Hence $G_{q}$ is defined over $k$. The lemma may be proved by étale base change on $Y$, and hence by Lemma 4.3 we may assume that $\mathcal{Y}=\left[V / G_{q}\right]$ for some smooth variety 
$V / k$ with a $k_{q}$-point $\widetilde{q}$ and an action of $G_{q}$ which fixes $\widetilde{q}$. The pullback of $\mathcal{W}$ to $V$ gives a $G_{q}$ equivariant étale cover $f: U \longrightarrow V$ such that $\mathcal{W}=\left[U / G_{q}\right]$. Let $\left\{p_{1}, \cdots, p_{d}\right\}=\mathcal{W} \times \mathcal{Y} \operatorname{Spec}\left(k_{q}\right)$ denote the set of $k_{q}$ points of $U$ lying over $\widetilde{q}$. Note that this is a finite set and moreover $d$ is exactly equal to the degree of the $\operatorname{map} \mathcal{W} \longrightarrow \mathcal{Y}$.

Now we claim that the following diagram is cartesian

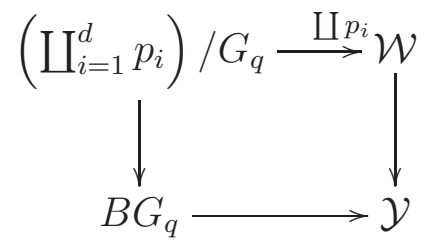

This claim follows from the fact that to check that above diagram is cartesian one further base change by the map $\operatorname{Spec}\left(k_{q}\right) \longrightarrow B G_{q}$. After this, the claim follows from the definition of the set $\left\{p_{1}, \cdots, p_{d}\right\}$.

Note that the left vertical arrow in the above diagram is a trivial étale cover if and only if the action of $G_{q}$ on $\left\{p_{1}, \cdots, p_{d}\right\}$ is trivial. More generally, we observe that the number of points in $W$ lying over the point $q$ of $Y$ is precisely equal to the set of orbits of $\left\{p_{1}, \cdots, p_{d}\right\}$ for $G_{q}$ action.

$(2) \Longrightarrow(1):$ If $W \longrightarrow Y$ is étale over $q$, then the number of elements lying over $q$ must match the generic degree (since it is also finite). Thus the number of orbits of $\left\{p_{1}, \cdots, p_{d}\right\}$ for $G_{q}$ action must be exactly $d$ which is equivalent to saying that the action of $G_{q}$ on $\left\{p_{1}, \cdots, p_{d}\right\}$ is trivial.

$(1) \Longrightarrow(2)$ : For any point $x \in U$, let $G_{x}$ be the stabilizer of $x$ for the action of $G_{q}$. Let $S$ be the set of points $x \in U$ such that $G_{x}=G_{f(x)}$, where $f$ denotes the map $U \longrightarrow V$.

We claim that $S$ is an open subset of $U$. To see this, consider the following commutative diagram

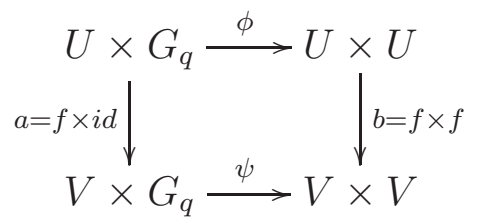

where $\phi$, and similarly $\psi$, is the map $(u, g) \longmapsto(u, g u)$. If

$$
\eta: U \times G_{q} \longrightarrow U
$$

is the projection, then the set $S$ is precisely the complement $\eta\left(a^{-1} \psi^{-1}\left(\Delta_{V}\right) \backslash \phi^{-1}\left(\Delta_{U}\right)\right)$, where $\Delta_{V}$ and $\Delta_{U}$ are the diagonals. Since $\eta$ is a closed map, and the diagonals $\Delta_{V}$ and $\Delta_{U}$ are closed, to prove the claim it suffices to show that $\phi^{-1}\left(\Delta_{U}\right)$ is open in $a^{-1} \psi^{-1}\left(\Delta_{V}\right)=\phi^{-1} b^{-1}\left(\Delta_{V}\right)$. Since $U \longrightarrow V$ is finite étale, the openness 
of $\phi^{-1}\left(\Delta_{U}\right)$ follows from the fact that $\Delta_{U}$ is a connected component of $b^{-1}\left(\Delta_{V}\right)$. This proves the claim that $S$ is open.

Let $T:=V \backslash f(U \backslash S)$. This $T$ is an open neighborhood of $q$ having the property that for every point $y^{\prime} \in U$ lying over a point $y$ in $T$, we have $G_{y}=G_{y^{\prime}}$.

Now, in order to prove that $(1) \Longrightarrow(2)$, we may replace $V$ by $T$, a $G_{q^{-}}$ invariant Zariski neighborhood of $q$. Thus, without loss of generality, we now assume that all points $y \in V$ have the property that for any point $y^{\prime} \in U$ with $f\left(y^{\prime}\right)=y$, we have $G_{y}=G_{f\left(y^{\prime}\right)}$. In other words, for every point $y$ of the stack $\mathcal{Y}$, the action of $G_{y}$ on the fiber of $\mathcal{W} \longrightarrow \mathcal{Y}$ is trivial. By Theorem 2.1 and Remark 2.2, the map $W \longrightarrow Y$ is étale.

Remark 2.8. We note that the only reason why one requires $\widetilde{X}$ to be smooth in Lemma 2.6] is to ensure purity, meaning any finite étale cover of an open subset of $X$, whose complement has codimension at least two, extends to a finite étale cover of $\widetilde{X}$. Thus the statement of Lemma 2.6 remains true if $\widetilde{X}$ is normal and satisfies purity.

\section{Group aCtions And Blow-ups}

The goal of this section is to prove Theorem 1.1. The proof uses the following observation on the tangent spaces to the points of a blow-up.

Proposition 3.1. Let $k$ be an algebraically closed field, and let $C$ be a cyclic group of prime power order acting faithfully on a smooth variety $X / k$. Assume that there exists a closed point $p \in X$ fixed by $C$. Then one can find a $C$ equivariant proper birational morphism $\pi: Y \longrightarrow X$ and a smooth closed point $\widetilde{p} \in Y$, such that the action of $C$ on the tangent space $T_{\widetilde{p}} Y$ is multiplication by a character of $C$.

Proof. Case (1): $|C|$ is a power of $C h a r(k)$. In this case, the group $C$ is unipotent. Let $V_{0} \subset T_{p} X$ be a maximal direct summand of the $C$-module $T_{p} X$ on which $C$ acts trivially. Thus we have a decomposition

$$
T_{p} X=V_{0} \oplus V^{\prime}
$$

Note that $V_{0}$ could be zero.

Using induction, we will show the following: by performing $C$-equivariant blow-ups at suitable closed points, $\operatorname{dim}_{k} V_{0}$ can be increased until it coincides with the dimension of $X$.

If $V^{\prime}=0$, then there is nothing to prove. Else, we can find a line $\ell \subset V^{\prime}$ on which the action of $C$ is trivial. Let $\widetilde{X} \longrightarrow X$ be the blow up of $X$ at $x_{0}$, and let $\widetilde{p} \in \widetilde{X}$ be the point above $p$ corresponding to the line $\ell$. The tangent space to $\widetilde{p}$ is canonically isomorphic to

$$
T_{\widetilde{p}} \widetilde{X}=\ell \oplus\left(\ell^{*} \otimes\left(T_{p} X / \ell\right)\right),
$$


where $\ell^{*}$ is the dual line. The group $C$ acts trivially on $\ell$ and hence also on its dual $\ell^{*}$. Therefore, we have a $C$-equivariant isomorphism

$$
T_{\widetilde{p}} \widetilde{X} \cong \ell \oplus V_{0} \oplus\left(V^{\prime} / \ell\right)
$$

Note that $C$ acts trivially on the above direct summand $\ell \oplus V_{0}$. Consequently, we have increased the dimension of the direct summand on which $C$ acts trivially, and now the proof of the claim is finished.

Case(2): $|C|$ is coprime to $C h a r(k)$. In this case, the group $C$ is linearly reductive, meaning every sub-representation of $C$ is a direct summand. Let $\chi$ be a primitive character (i.e., a generator of the character group) of $C$. For any integer $a$, let

$$
V_{\chi^{a}} \subset T_{p} X
$$

be the isotypical component on which $C$ acts as multiplication through $\chi^{a}$. There are finitely many integers $\left\{a_{i}\right\}_{i=1}^{r}$ such that

$$
T_{p} X=\bigoplus_{i=1}^{r} V_{\chi^{a_{i}}}
$$

Since the action of $C$ on $T_{p} X$ is faithful, at least one $a_{i}$ (say $a_{1}$ ) is coprime to $|C|$. As $|C|$ is a prime power, we conclude that $\chi^{a_{1}}$ is also a primitive character. By replacing $\chi$ by $\chi^{a_{1}}$, we may assume that $a_{1}=1$.

If $r=1$, then there is nothing to prove. We may therefore assume that $r \geq 2$. Let $\ell \subset V_{\chi}=V_{\chi^{1}}$ be a line, and write

$$
V_{\chi}=\ell \oplus V_{\chi}^{\prime}
$$

By Lemma 3.2, there exists a closed subvariety $Y \subset X$ containing $p$, and smooth at $p$, such that $T_{p} Y=V_{\chi}^{\prime}$. Let

$$
\pi_{1}: X_{1} \longrightarrow X
$$

be the blow up of $X$ along $Y$. Let $p_{1} \in X_{1}$ be the point lying above $p$ corresponding to the normal direction $\ell$. By Lemma 3.3, we have

$$
T_{p_{1}} X_{1}=V_{\chi} \oplus \ell^{*} \otimes\left(\bigoplus_{i=2}^{r} V_{\chi^{a_{i}}}\right) \text {. }
$$

Since $V_{\chi}$ and hence $\ell$ are naturally subspaces of $T_{p_{1}} X_{1}$, we can inductively define $X_{m}, m \geq 1$, as the blow-up of $X_{m-1}$ along the subvariety through $p_{m-1}$ defined by $V_{\chi}^{\prime} \subset T_{p_{m-1}} X_{m-1}$ (see Lemma 3.3) with $p_{m}$ being the point lying above $p_{m-1}$ corresponding to the normal direction $\ell$. Thus the tangent space at $p_{m}$ decomposes as

$$
T_{p_{m}} X_{m}=V_{\chi} \oplus\left(\bigoplus_{i=2}^{r} V_{\chi^{a_{i}-m}}\right) .
$$


Therefore, if $m$ is an integer such that

$$
a_{2}-m \equiv 1 \bmod |C|,
$$

we see that the number of characters of $C$ which have non-zero eigenspaces in $T_{p_{m}} X_{m}$ is strictly less than $r$. Now, by induction, we may replace $X$ with a higher birational model and assume that $r=1$. This completes the proof of the proposition.

Lemma 3.2. Continuing with the notation of Proposition 3.1, assume that Char $(k)$ does not divide $|C|$. Then given any $C$-invariant subspace $W \subset T_{p}(X)$, there exists a $C$-invariant closed subvariety $Y \subset X$, containing $p$ and smooth at $p$, such that $W=T_{p} Y$.

Proof. Since $C$ is linearly reductive, we can find a $C$-equivariant decomposition

$$
T_{p} X=W \oplus W_{1}
$$

This gives a $C$-equivariant decomposition of the cotangent space

$$
m_{p} / m_{p}^{2}=\left(T_{p} X\right)^{*}=W^{*} \oplus W_{1}^{*},
$$

where $m_{p}$ is the maximal ideal of the local ring $\mathcal{O}_{X, p}$ at $p$. In order to prove the lemma we may assume that $X=\operatorname{Spec}(A)$ is an affine scheme, by looking at a $C$-equivariant affine neighborhood of $p$.

The maximal ideal of $A$ corresponding to $p$ will also be denoted by $m_{p}$; the distinction will be clear from the context. We have a surjection

$$
A \longrightarrow A / m_{p}^{2}
$$

This is a $C$-equivariant homomorphism and it admits a $C$-equivariant splitting. Therefore, if $r=\operatorname{codim}_{k(p)} W$, then there exist $f_{1}, \cdots, f_{r} \in A$ such that the $k$-span of $\left\{f_{i}\right\}_{i=1}^{r}$ is $C$-invariant and it maps isomorphically onto $W_{1}^{*}$. Now let $Y$ be the closed subvariety defined by the ideal generated by $\left\{f_{i}\right\}_{i=1}^{r}$. If $m_{p, Y}$ is the ideal of the local ring of $p$ at $Y$, then

$$
m_{p, Y} / m_{p, Y}^{2}=W^{*} .
$$

Taking duals, we see that $T_{p} Y=W$ as required. To show $Y$ is smooth at $p$, we first note that by Krull's principal ideal theorem, we have $\operatorname{codim}(Y) \leq r$. However, $\operatorname{dim}_{k(p)}\left(m_{p, Y} / m_{p, Y}^{2}\right)=r$. This completes the proof.

Lemma 3.3. Let $G$ be a finite group acting on $X$ with a closed fixed point $p$. Assume that $|G|$ is coprime to Char $(k)$. Let $Y \subset X$ be a closed, smooth, $G-$ invariant subvariety containing $p$, and let

$$
\pi: \tilde{X} \longrightarrow X
$$

be the blow-up of $X$ along $Y$. Take a line $\ell \subset T_{p} X$ not contained in $T_{p} Y$. Let $\widetilde{p} \in \tilde{X}$ be the point above $p$ corresponding to the normal direction given by $\ell$. Then there exists a $G$-equivariant isomorphism

$$
T_{\widetilde{p}} \widetilde{X} \cong\left(T_{p} Y \oplus \ell\right) \oplus\left(\ell^{*} \otimes\left(T_{p} X /\left(T_{p} Y+\ell\right)\right)\right) .
$$


Proof. We observe that the assertion depends only on a formal neighborhood of $p$ in $X$. The strategy of the proof consists of the following two steps:

- Show that formally locally at $p$, the triple $(X, Y, p)$ is $G$ equivariantly isomorphic to $\left(\mathbb{A}_{k}^{n}, \mathbb{A}_{k}^{m}, \underline{0}\right)$, where $G$ acts linearly on $\mathbb{A}_{k}^{n}$ with $\mathbb{A}_{k}^{m} \subset \mathbb{A}_{k}^{n}$ being a $G$-invariant subspace, and

- check the claim of the lemma by using the explicit construction of the blow up as a closed subvariety of $\mathbb{A}_{k}^{n} \times_{k} \mathbb{P}_{k}^{n-m-1}$.

The later step is straightforward and we omit the details.

Without loss of any generality, we assume that $X=\operatorname{Spec}(A)$ is affine. The maximal ideal corresponding to $p$ will be denoted by $m_{p}$. Define

$$
V:=m_{p} / m_{p}^{2} \text { and } W:=m_{p, Y} / m_{p, Y}^{2} \subset V,
$$

where $m_{p, Y}$ is the maximal ideal of $p$ in the local ring of $Y$. Since $G$ is linearly reductive, there exists a $G$-equivariant splitting of the epimorphism

$$
A \longrightarrow A / m_{p}^{2} \text {. }
$$

Thus we get an $n$-dimensional $k$-subspace $V_{0} \subset A$ that maps isomorphically onto $V$. We denote by $W_{0}$ this subspace of $V_{0}$ mapping isomorphically onto $W$. The canonical $k$-algebra homomorphism

$$
\operatorname{Sym}_{k}^{*}\left(V_{0}\right) \longrightarrow A
$$

gives the formal local isomorphism with the affine space $\operatorname{Spec}\left(\mathcal{S} y m^{*}\left(V_{0}\right)\right)$ that we are seeking.

Proof of Theorem 1.1. In order to prove the theorem, we may replace $k$ by its algebraic closure inside the function field of $X$, and assume that $X$, and hence $\widetilde{X}$, are geometrically connected. Fix a separable closure $K / k$, and let $X_{K}$ (respectively, $\widetilde{X}_{K}$ ) denote the base extension to $K$ of $X$ (respectively, $\widetilde{X}$ ). We lift the geometric point $x_{0}$ to the corresponding base extended varieties. Therefore, we have a commutative diagram with exact rows:

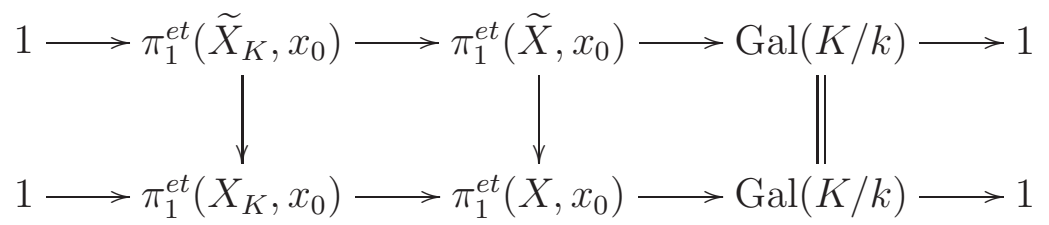

It is now clear that to prove that the middle vertical arrow is an isomorphism, it is enough to prove that the left-most vertical arrow is an isomorphism. Thus we may base extend to $K$ and assume that our field is separably closed. Further, if $\bar{K} / K$ is an algebraic closure of $K$, then as $K$ is separably closed, this extension is purely inseparable. Base extension by an inseparable extension does not change the étale fundamental group. Hence we may base extend to the algebraic closure of $K$ and henceforth assume that $K$ is in fact an algebraically closed field. 
The theorem is equivalent to the statement that the pullback functor

$$
\operatorname{ET}(X) \longrightarrow \operatorname{ET}(\widetilde{X})
$$

(see Notation 2.3) is an equivalence of categories. This question is étale local on $X$ and hence we may assume that $X$ is actually a quotient of a variety $Y$ by a finite group $G$ acting faithfully on $Y$. Let

$$
\mathcal{X}=[Y / G]
$$

be the quotient stack. Fix a point $x_{0}$ of $\mathcal{X}$. By Theorem 2.1 and Lemma 2.6, it is enough to show that the natural inclusion

$$
\widetilde{N}_{x_{0}} \subset N_{x_{0}}
$$

(see Notation 2.3) is surjective. This is equivalent to the statement that for any point $p$ of $\mathcal{X}$, the images of $\phi_{q, \mathcal{X}}($ see $(2.3))$ for all $(\mathcal{Y}, q) \in \Sigma_{\mathcal{X}}$ such that $q$ lies over $p$, together generate the isotropy group $G_{p}$ at $p$. We will prove this statement by showing that all elements of $G_{p}$ of prime power order are in the image of $\phi_{q, \mathcal{X}}$ for some $(\mathcal{Y}, q)$.

Let $x \in G_{p}$ be an element of prime power order, and let $C$ be the cyclic subgroup of $G_{p}$ generated by $x$. It suffices to find a $(\mathcal{Y}, q) \in \Sigma_{\mathcal{X}}$ (see Notation 2.3) such that image of the induced homomorphism on isotropy groups

$$
G_{q} \longrightarrow G_{p}
$$

is $C$. By Lemma 3.3, there exists a $C$-equivariant proper birational morphism $\pi: \widetilde{Y} \longrightarrow Y$ and a point $\widetilde{p} \in \widetilde{Y}$ with $\pi(\widetilde{p})=p$, such that the action of $C$ on the tangent space $T_{\widetilde{p}} \widetilde{Y}$ is multiplication by a character.

Let $Z \longrightarrow \widetilde{Y}$ be the blow-up at $\widetilde{p}$, and let $q$ denote the generic point of the exceptional divisor for this blow-up of $\widetilde{Y}$. Then clearly $C$ acts trivially on this exceptional divisor, and hence the isotropy group of $q$ is $C$. For every $g \in G$, define $g^{*} Z$ by the following Cartesian diagram

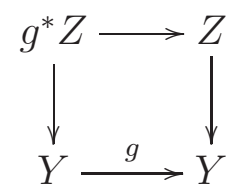

Each $g^{*} Z$ is a $Y$-scheme. Consider the product

$$
P=\prod_{g \in G} g^{*} Z
$$

in the category of $Y$-schemes, i.e., it is a fiber product over $Y$.

We have a natural action of the group $G$ on $P$ which permutes the factors. Since each map $g^{*} Z \longrightarrow Y$ is proper and birational, there exists a unique dominant irreducible component of $P$. We denote the normalization of this component 
by $T$. By construction, $\pi_{T}: T \longrightarrow Y$ is a $G$-equivariant proper birational morphism.

Let $f: T \longrightarrow Z$ be the restriction of the natural projection map

$$
P \longrightarrow e^{*} Z=Z
$$

where $e \in G$ is the identity element. We claim that $f$ is $C$-equivariant.

To prove this claim, we first observe that there exists a $G$-invariant open subset $U$ of $Y$ over which both

$$
\pi_{T}: T \longrightarrow Y \text { and } \pi_{Z}: Z \longrightarrow Y
$$

are isomorphisms. Clearly, the restriction of $f: T \longrightarrow Z$ to $U$ is $C$-equivariant. But $T$ is a separated integral variety, and $U$ is dense in $T$. Consequently, $f$ is also $C$-equivariant, proving the claim.

Since $Z$ is normal, the morphism $f$ is an isomorphism over all codimension one points of $Z$, in particular over $q$. Recall that $q$ is the codimension one point of $Z$ whose isotropy group is whole of $C$. Hence there exists a unique codimension one point $\widetilde{q}$ of $T$ lying over $q$. Since $T \longrightarrow Z$ is $C$-equivariant, we conclude that $C$ is contained in the isotropy group of $q$. This completes the proof of Theorem 1.1 .

Remark 3.4. In view of Remark 2.8, the statement of Theorem 1.1 continues to hold for all normal varieties $\widetilde{X}$ which satisfy purity.

Below we mention Kollár's observation which handles the general case of quotient of a smooth variety by a finite group scheme. The argument to deduce the theorem below from Theorem 1.1 was outlined to us by Kollár in Ko2]. The proof given below is based on a significant simplification of this argument given by the referee.

Theorem 3.5 ([Ko2]). Let $k$ be any field, and let $G / k$ be a finite group scheme acting faithfully on a smooth variety $Y$. If $X$ is the geometric quotient of $Y$ by $G$, and $f: \widetilde{X} \longrightarrow X$ is any proper birational morphism with $\widetilde{X}$ smooth, then the homomorphism

$$
\pi_{1}^{e t}(\tilde{X}, x) \longrightarrow \pi_{1}^{e t}(X, f(x))
$$

is an isomorphism for any geometric point $x$ of $\widetilde{X}$.

Proof. Without any loss of generality we may assume that $k$ is perfect (or even algebraically closed) by base extending to the algebraic closure of $k$. Thus the reduced subscheme $G_{\text {red }} \subset G$ is a subgroup.

Consider the quotient stack $\mathcal{X}:=\left[Y / G_{\text {red }}\right]$. Let $\mathcal{X} \longrightarrow X^{\prime}$ be its coarse moduli space. Take $\widetilde{X}$ as in the theorem. Let $\widetilde{X}^{\prime}$ be the normalization of $\widetilde{X}$ in the function field of $X^{\prime}$. We thus have the following commutative diagram 
where horizontal arrows are proper birational and vertical arrows are universal homeomorphisms.

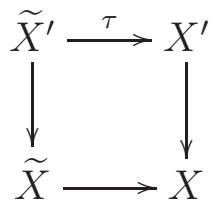

Thus in order to prove the theorem, it is enough to show that the induced homomorphism

$$
\tau_{*}: \pi_{1}^{e t}\left(\tilde{X}^{\prime}, z\right) \longrightarrow \pi_{1}^{e t}\left(X^{\prime}, \tau(z)\right) .
$$

We note that $\widetilde{X}^{\prime}$ is a normal variety satisfying purity because $\widetilde{X}$ is smooth (see Remark 2.8). Now from Theorem 1.1 and Remark 3.4 it follows that $\tau_{*}$ is an isomorphism.

\section{Fundamental group of the symmetric PRoduCt}

Let $S^{n}$ be the group of permutations of $\{1, \cdots, n\}$. The following lemma will be used to prove Theorem 1.2.

Lemma 4.1. Let $G$ be any group, and $G^{n}:=\prod_{i=1}^{n} G$. For the natural action on $G^{n}$ of $S^{n}$, consider the semi-direct product $G^{n} \rtimes S^{n}$. Let $N$ be the normal subgroup of $G^{n} \rtimes S^{n}$ generated by the image of $S^{n}$ for the natural injection $S^{n} \longrightarrow G^{n} \rtimes S^{n}$. Then the quotient $\left(G^{n} \rtimes S^{n}\right) / N$ is canonically isomorphic to $G^{a b}:=G /[G, G]$, the abelianization of $G$.

Proof. The group operation of $G^{n} \rtimes S^{n}$ is given by

$$
\left(g_{1}, \cdots, g_{n}, \sigma\right) \cdot\left(g_{1}^{\prime}, \cdots, g_{n}^{\prime}, \sigma^{\prime}\right)=\left(g_{1} g_{\sigma(1)}, \cdots, g_{n} g_{\sigma(n)}, \sigma \sigma^{\prime}\right) .
$$

We have the following relation modulo $N$ :

$$
\left(g_{1}, \cdots, g_{n}, \gamma\right)=\left(g_{\sigma(1)}, \cdots, g_{\sigma(n)}, \gamma\right) \bmod N, \forall \sigma, \gamma \in S^{n} .
$$

Thus

$$
\begin{aligned}
\left(g_{1}, \cdots, g_{n}, \sigma\right)= & \prod_{i=1}^{n}\left(1, \cdots, g_{i}, \cdots, 1, \sigma\right)=\prod_{i=1}^{n}\left(g_{i}, 1, \cdots, 1, \sigma\right) \\
& =\left(g_{1} \cdots g_{n}, 1, \cdots, 1, \sigma\right) .
\end{aligned}
$$

Also, since

$$
(g, 1, \cdots, 1, \sigma) \cdot(h, 1, \cdots, \sigma)=(g h, 1, \cdots, 1, \sigma)=(h g, 1, \cdots, 1, \sigma),
$$

one can deduce that

$$
(g, 1, \cdots, 1, \sigma)=1 \bmod N
$$

if $g$ belongs to the commutator subgroup $[G, G] \subset G$. Define the homomorphism

$$
\phi: G^{n} \rtimes S^{n} \longrightarrow G^{a b},\left(g_{1}, \cdots, g_{n}, \sigma\right) \longmapsto \overline{g_{1}} \cdots \overline{g_{n}} .
$$

Clearly $N \subset \operatorname{kernel}(\phi)$. 
We claim that $N=\operatorname{kernel}(\phi)$. To prove this claim, take any $\left(g_{1}, \cdots, g_{n}, \sigma\right) \in$ $\operatorname{kernel}(\phi)$. By the definition of $\phi$,

$$
\prod_{i=1}^{n} g_{i} \in[G, G]
$$

From (4.1) and (4.2),

$$
\left(g_{1}, \cdots, g_{n}, \sigma\right)=\left(\prod_{i=1}^{n} g_{i}, 1, \cdots, 1, \sigma\right)=1 \bmod N
$$

This proves the claim. The claim completes the proof of the lemma.

Henceforth, we assume that the field $k$ is algebraically closed.

Lemma 4.2. Let $X$ be a a proper, integral $k$ variety. Let $\mathcal{X}:=\left[X^{n} / S^{n}\right]$ for the natural permutation action of $S^{n}$ on $X^{n}$. Let $x$ be a geometric point of $\mathcal{X}$ of the form

$$
x=\left(x_{0}, \cdots, x_{0}\right)
$$

where $x_{0}$ is a geometric point of $X$. Then $\pi_{1}^{\text {et }}(\mathcal{X}, x)$ is canonically isomorphic to the semi-direct product $\pi_{1}^{\text {et }}\left(X, x_{0}\right)^{n} \rtimes S^{n}$ for the natural action of $S^{n}$ on $\pi_{1}^{e t}\left(X, x_{0}\right)^{n}$.

Proof. The group $\pi_{1}^{e t}\left(X^{n}, x\right)$ is canonically identified with $\pi_{1}^{e t}\left(X, x_{0}\right)^{n}$ because $X$ is proper and integral (see [SGA, X.1.3 and X.1.7]). Since the quotient map $X^{n} \longrightarrow \mathcal{X}$ is a Galois étale cover with Galois group $S^{n}$, we have a short exact sequence

$$
1 \longrightarrow \pi_{1}^{e t}\left(X, x_{0}\right)^{n} \longrightarrow \pi_{1}(\mathcal{X}, x) \longrightarrow S^{n} \longrightarrow 1
$$

Moreover, the isotropy group of the point $x$ in $X^{n}$ is precisely $S^{n}$, thus yielding a representable 1-morphism

$$
\left[x / S^{n}\right] \longrightarrow \mathcal{X}
$$

which induces a right splitting

$$
\pi_{1}^{e t}\left(\left[x / S^{n}\right], x\right)=S^{n} \stackrel{\theta}{\longrightarrow} \pi_{1}^{e t}(\mathcal{X}, x)
$$

of the above short exact sequence.

It remains to show that for any $\sigma \in S^{n}$, the conjugation $\pi_{1}^{e t}(X, x)^{n}$ by $\theta(\sigma)$ coincides with the natural action of $\sigma$ induced by permuting the factors. To show that two given elements of $\pi_{1}^{e t}(\mathcal{X}, x)$ coincide, it is enough to show that their actions on the geometric fibers over $x$ of Galois étale covers $\mathcal{Y} \longrightarrow \mathcal{X}$ agree. In fact it suffices to consider only Galois étale covers

$$
\tilde{f}: \mathcal{Y}=Y^{n} \longrightarrow \mathcal{X}
$$

which are compositions of the form

$$
Y^{n} \stackrel{(f, \cdots, f)}{\longrightarrow} X^{n} \longrightarrow \mathcal{X}
$$


where $f: Y \longrightarrow X$ is a Galois étale cover, because these covers $\tilde{f}$ are cofinal in the category of Galois étale covers of $\mathcal{X}$; here $X^{n} \longrightarrow \mathcal{X}$ is the quotient map. Let $f^{-1}(x)$ denote the fiber of $f$ over the point $x$. Then $\widetilde{f}^{-1}(x)=f^{-1}(x)^{n}$. We note that the action of $S^{n}$ via $\theta$ on $f^{-1}(x)^{n}$ is simply the natural permutation action. Indeed, this can be deduced from the fact that the following diagram is Cartesian

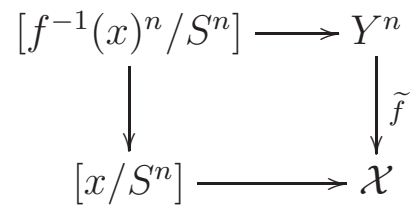

where $\left[f^{-1}(x)^{n} / S^{n}\right]$ is the stack quotient by the permutation action of $S^{n}$ on $f^{-1}(x)^{n}$. Now if

$$
\widetilde{\phi}=\left(\phi_{1}, \cdots, \phi_{n}\right) \in \operatorname{Gal}\left(Y^{n} / X^{n}\right),
$$

then for $\sigma \in S^{n}$, the equality

$$
\theta(\sigma) \cdot \tilde{\phi} \cdot \theta(\sigma)^{-1}=\left(\phi_{\sigma^{-1}(1)}, \cdots, \phi_{\sigma^{-1}(n)}\right)
$$

can be deduced by comparing their actions on $f^{-1}(x)^{n}$. This proves the claim that the action of $S^{n}$ on $\pi_{1}^{e t}\left(X^{n}, x\right)$ is the same as the one induced by the permutation action of $S^{n}$ on $X^{n}$.

Lemma 4.3. Let $\mathcal{X} / k$ be a separated Deligne-Mumford stack over a noetherian base scheme and $\pi: \mathcal{X} \longrightarrow X$ be its coarse moduli space. Then étale locally around any point $p$ of $X$, the stack $\mathcal{X}$ can be written as a quotient of a $k$-scheme by the isotropy group of $\mathcal{X}$ at $p$.

Proof. By [AV, Lemma 2.2.3], we may assume that $\mathcal{X}=[V / G]$, where $G$ is a finite group acting on a scheme $V$. Let $\widetilde{p}$ be a point of $V$ lying over $p$, and let $G_{\widetilde{p}} \subset G$ be the isotropy group of $\widetilde{p}$. The group $G_{\widetilde{p}}$ is canonically identified with the isotropy group $G_{p}$ of the stack $\mathcal{X}$ at the point $p$.

We claim that there exists a Zariski open neighborhood of $\widetilde{p}$ in $V$, such that the isotropy group of all points in this neighborhood is contained in $G_{\widetilde{p}}$.

To prove the above claim, note that for any $g \in G$, the fixed locus $Z_{g}$ of $g$ is a closed subscheme of $V$. We now simply take this neighborhood to be the complement of $\bigcup_{g \in G_{\widetilde{p}}} Z_{g}$.

We now replace $V$ by this neighborhood and assume that $V$ itself has the property that all isotropy groups are contained in $G_{\widetilde{p}}$. This ensures that the map $\left[V / G_{\widetilde{p}}\right] \longrightarrow[V / G]=\mathcal{X}$, as well as the induced map on coarse moduli spaces is finite étale. Thus by Remark 2.2, the stack $\left[V / G_{\widetilde{p}}\right]$ is pullback of a finite étale cover of the coarse moduli space of $\mathcal{X}$. In other words, étale locally on the coarse moduli space, $\mathcal{X}$ of the type $\left[V / G_{\widetilde{p}}\right]$, as required.

Notation 4.4 (Specialization map on isotropy groups). Let $\mathcal{X} / k$ be a separated Deligne-Mumford stack. Then for any geometric point $q$ of $\mathcal{X}$, specializing to a 
geometric point $p$, one can produce a homomorphism

$$
\phi_{q, p}: G_{q} \longrightarrow G_{p}
$$

which is well defined up to conjugation by an element of $G_{p}$; we will recall its construction. To define $\phi_{q, p}$, we may work étale locally on the coarse moduli space of $\mathcal{X}$ and by Lemma 4.3 assume that $\mathcal{X}$ is the quotient $\left[V / G_{p}\right]$, where $G_{p}$ acts on $V$ with a fixed point $\widetilde{p}$ lying over $p$. Since the point $q$ of $\mathcal{X}$ specializes to $p$, there exists a point $\widetilde{q}$ (not necessarily unique) of $V$, lying over $q$ which specializes to $\widetilde{p}$. As $\widetilde{q}$ specializes to $\widetilde{p}$, the isotropy group $G_{\widetilde{q}}$ is a subgroup of the isotropy group $G_{\widetilde{p}}$ of $p$. However, we also have natural identifications $G_{\widetilde{p}}=G_{p}$ and $G_{\widetilde{q}}=G_{q}$. This gives rise to an inclusion $\phi_{q, p}: G_{q} \longrightarrow G_{p}$. Note however, a different choice of $\widetilde{q}$ leads to another homomorphism $G_{q} \longrightarrow G_{p}$ which differs from the above choice by an inner automorphism of $G_{p}$. Thus $\phi_{q, p}$ is well defined up to an inner automorphism.

Lemma 4.5. Let $p, q$ be two geometric points of a connected separated DeligneMumford stack $\mathcal{X}$ with $q$ specializing to $p$. Let $x$ be any other geometric point of $\mathcal{X}$. Then the following diagram commutes up to an inner automorphism of $\pi_{1}^{e t}(\mathcal{X}, x)$ :

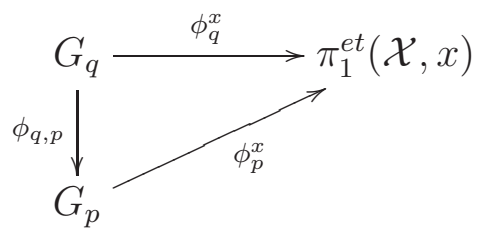

(see (4.3) and (2.2) for the homomorphisms).

Proof. Without loss of generality we may assume that $x=p$ and hence $\phi_{p}^{x}=\phi_{p}$, where $\phi_{p}$ is the canonical homomorphism from $G_{p}$ to $\pi_{1}^{e t}(\mathcal{X}, p)$ induced by

$$
\left[p / G_{p}\right] \longrightarrow \mathcal{X}
$$

Let $V, \widetilde{p}$ and $\widetilde{q}$ be as in Notation 4.4, We denote by $\widetilde{V}$ the strict henselization of $V$ at $\widetilde{p}$ and lift points $\widetilde{p}$ and $\widetilde{q}$ to points of $\widetilde{V}$, which are also denoted by $\widetilde{p}, \widetilde{q}$ for ease of notation. The morphisms $\left[p / G_{p}\right] \longrightarrow \mathcal{X}$ and $\left[q / G_{q}\right] \longrightarrow \mathcal{X}$ factor through $\left[\widetilde{V} / G_{p}\right]$. Therefore, to prove the lemma, we may replace $\mathcal{X}$ by the stack $\left[\widetilde{V} / G_{q}\right]$. Since $\widetilde{V}$ is strict henselian, the homomorphism

$$
G_{p} \longrightarrow \pi_{1}^{e t}\left(\left[\widetilde{V} / G_{p}\right], p\right)
$$

is a bijection. Thus it remains to show that the homomorphism

$$
\phi_{q}^{p}: G_{q} \longrightarrow \pi_{1}^{e t}\left(\left[\widetilde{V} / G_{p}\right], p\right)
$$

defined in (2.2), coincides, up to an inner automorphism, with the inclusion of isotropy groups $G_{q} \hookrightarrow G_{p}$. However, this follows easily by comparing the actions of $G_{q}$ and $G_{p}$ on the geometric fibers of the universal cover $\widetilde{V} \longrightarrow\left[\widetilde{V} / G_{p}\right]$. 
Proof of Theorem 1.2. Let $\mathcal{X}=\left[X^{n} / S^{n}\right]$, so that $\pi: \mathcal{X} \longrightarrow \operatorname{Sym}^{n}(X)$ is its coarse moduli space. Let $x$ and $x_{0}$ be as in the statement of the theorem. We think of $x$ also as a geometric point of both $\mathcal{X}$ and $X^{n}$. The quotient map

$$
f: X^{n} \longrightarrow \mathcal{X}
$$

is a Galois étale cover with Galois group $S^{n}$. By Lemma 4.2, we have

$$
\pi_{1}^{e t}(\mathcal{X}, x)=\pi_{1}^{e t}\left(X, x_{0}\right)^{n} \rtimes S^{n} .
$$

Moreover, the map

$$
\left[x / S^{n}\right] \longrightarrow \mathcal{X}
$$

induces a section $t: S^{n} \longrightarrow \pi_{1}^{e t}(\mathcal{X}, x)$ of $\pi_{1}^{e t}(\mathcal{X}, x) \longrightarrow S^{n}$. The image $t\left(S^{n}\right)$ coincides with the image of the homomorphism

$$
G_{x}=S^{n} \longrightarrow \pi_{1}^{e t}(\mathcal{X}, x)
$$

in (2.1).

Using notation from Section 2, we claim that the closed normal subgroup of $\pi_{1}^{e t}(\mathcal{X}, x)$ generated by image of $t$ is entire $N_{x}$ (see Theorem 2.1). By definition of $N_{x}$, to prove the claim it is enough to show that given any other point $p$, the image of

$$
G_{p} \longrightarrow \pi_{1}^{e t}(\mathcal{X}, x)
$$

is contained inside the image of $t$. Now, lift $p$ to a geometric point of $X^{n}$, and let $I \subset\{1, \cdots, n\}$ be the maximal subset such that the coordinates of $p$ indexed by $I$ are equal. We have the subvariety $X_{I}^{n} \subset X^{n}$ defined by the condition that all coordinates indexed by $I$ are equal. Let $q$ be the generic point of $X_{I}^{n}$. Then $q$ specializes to $p$ and the inclusion $G_{q} \hookrightarrow G_{p}$ is an isomorphism. But $q$ also specializes to $x$. The theorem now follows from Lemma 4.5 .

\section{REFERENCES}

[AV] D. Abramovich and A. Vistoli, Compactifying the space of stable maps, Jour. Amer. Math. Soc. 15 (2002), 27-75.

[DT] A. Dold and R. Thom, Quasifaserungen und unendliche symmetrische Produkte, Ann. of Math. 67 (1958), 239-281.

[Fo] J. Fogarty, Algebraic families on an algebraic surface, Amer. Jour. Math. 90 (1968), $511-521$.

[EGA] Grothendieck, A. Eléments de géométrie algébrique. IV. Étude locale des schémas et des morphismes de schémas. III. Inst. Hautes Études Sci. Publ. Math. No. 281966 255 pp.

[SGA] A. Grothendieck and M. Raynaud, Revêtements étales et groupe fondamental (SGA 1). Séminaire de géométrie algébrique du Bois Marie 1960-61, Lect. Notes Math. 224, Springer, Berlin, arXiv:math/0206203 [math.AG].

[Ko1] J. Kollár, Shafarevich maps and plurigenera of algebraic varieties, Invent. Math. 113 (1993), 177-215.

[Ko2] J. Kollár, private communication via email, June 14, 2013.

[No] B. Noohi, Fundamental groups of algebraic stacks, Jour. Inst. Math. Jussieu 3 (2004), 69-103. 
[KT] S. Kallel and W. Taamallah, The geometry and fundamental group of permutation products and fat diagonals, www-gat.univ-lille1.fr/ kallel/Papers/perprod2.pdf.

School of Mathematics, Tata Institute of Fundamental Research, Homi Bhabha ROAD, BOMBAY 400005, INDiA

E-mail address: indranil@math.tifr.res.in

School of Mathematics, Tata Institute of Fundamental Research, Homi Bhabha ROAD, BOMBAY 400005, INDIA

E-mail address: amit@math.tifr.res.in 